\title{
Europäische Perspektiven
}

Der Zusammenschluss von bislang 28 Staaten in der Europäischen Union ist verbunden mit einer Politik des Friedens, die mit dem ersten Entwurf einer europäischen Gemeinschaft von Jean Monnet, Robert Schuman, Alcide de Gasperi, Paul Henri Spaak und Konrad Adenauer in den 50er Jahren des 20. Jahrhunderts eingeleitet wurde. Sie kann als Renaissance der europäischen Friedensidee betrachtet werden, die unter Karl dem Großen ihren Ausgang nahm. Die Verwirklichung von „Frieden und Gerechtigkeit“ im Sinne des biblischen Psalms $(85,11-14)$ sah der Kaiser am Ende seines Lebens als dringlichste Aufgabe. Insbesondere Deutschland und Frankreich erinnern sich in diesem Jahr an den 1200. Todestag von Karl dem Großen, der die Notwendigkeit „der kulturellen und intellektuellen Erneuerung mit Hilfe von Fremden" (Johannes Fried) betonte. Die Enkel des mittelalterlichen Kaisers haben die Idee der innereuropäischen Versöhnung in den „Eiden von Straßburg“ 842 in den Sprachen Deutsch und Französisch formuliert und damit einen Meilenstein für ein friedliches Europa gesetzt. Maßlose Gewalt und Zerstörungswut haben in zwei Weltkriegen Europa zerstört. Im Erschrecken darüber, was Menschen sich antun können, forderte der Politikwissenschaftler Dolf Sternberger 1960 in seiner Antrittsvorlesung den „Frieden als das einzige Ziel der Politik“ und die „Politik als einzigen Weg zum Frieden“. Für Europa bedeutet diese Forderung eine prinzipielle Wende zum Rechtsstaat verbunden mit dem Bekenntnis zu unveräußerlichen Menschenrechten als Grundlage der menschlichen Gesellschaft.

Trotz Rückläufen und Turbulenzen im europäischen Einigungsprozess ist eine EU-Grundrechtecharta als Teil des Primärrechts mit dem Reformvertrag von Lissabon 2007 rechtswirksam geworden. Vor dem Hintergrund dieser gesamteuropäischen Leistung zeichnen sich neue Entwicklungen in einer „hinreichend demokratischen Verrechtlichung" (Jürgen Habermas) ab. Das betrifft insbesondere die unionsrechtliche Gewährleistung eines der elementarsten Grund- und Menschenrechte im digitalen 21. Jahrhundert: das Recht auf Privatsphäre und Da- tenschutz. In einem wegweisenden Urteil hat der EuGH am 8. April $2014^{1}$ ein grundsätzliches Verbot der Vorratsdatenspeicherung ausgesprochen und damit erstmals eine entscheidende Rolle im europäischen Menschenrechtsschutz der EU übernommen, die bisher eher vom EGMR wahrgenommen wurde. Die EU plant eine allgemeine Datenschutzgrundverordnung und eine spezielle Richtlinie für die Bereiche Justiz und Inneres. Die Kommission und das Europäische Parlament stimmen bei der Verhandlung der DSGVO weitgehend überein. Eine offizielle Stellungnahme des Rates zu der Verordnung, die bis Ende 2015 / Mitte 2016. verabschiedet werden soll, steht noch aus. Falls Kommission und Parlament an der Paketlösung („Verordnung nur bei gleichzeitiger Verabschiedung der Richtlinie“) festhalten, wird der Zeitpunkt der Einigung möglicherweise später sein, weil die Richtlinie noch verhandelt werden muss.

Nach den Enthüllungen des Whistleblowers Snowden ist die Durchsetzung eines europäischen Datenschutzes ein „Muss“, um der Gefahr des Verlustes eines elementaren Rechtsguts zu begegnen. James Bamford warnte schon 2013 in diesem Zusammenhang: „There is the abyss from which there is no return." Im Sinne des uralten Gebots von Frieden und Gerechtigkeit, das in Art. 1 Abs. 1 GG im Kontext des Schutzes der Menschenrechte aufscheint, muss sich der europäische Citoyen auf den Weg machen und sich dabei für einen Datenschutz in der EU gemäß dem verfassungsmäßigen Verhältnismäßigkeitsprinzip als goldene Regel einsetzen. Auf dem Weg zu einer menschengerechten Datenschutzregel entscheiden Vertreter vielegestaltiger europäischer Rechtskulturen und Sprachen, die sich annähern und nach dem Grundsatz der Fairness, der Chancengleichheit also, aufeinander zugehen.

1 Urteil in den verbundenen Rechtssachen C-293/12 und C-594/12 vom 8. April 2014, DuD 2014, 488; siehe dazu auch den Beitrag von Leutheusser-Schnarrenberger in diesem Heft. 